\title{
Prevalence of plasmid-mediated quinolone-resistance determinants in Shigella flexneri isolates from Anhui Province, China
}

\author{
Zizhong Xiong ${ }^{1,2}$, Jun $\mathrm{Li}^{2}$, Tao $\mathrm{Li}^{3}$, Jilu Shen ${ }^{3}$, Fupin $\mathrm{Hu}^{4}$ and Minggui Wang ${ }^{4}$
}

Quinolones are used extensively to treat Shigella flexneri infection, and plasmid-mediated quinolone resistance (PMQR) determinants were recently reported. A total of $26 \mathrm{~S}$. flexneri isolates collected from Anhui province, China, in 2005 were screened for PMQR determinants, bla gene, gyrA and parC genes, by PCR and sequencing. PMQR determinants were present in 53.8\% (14 of 26) of isolates and $q n r A_{1}, q n r S_{1}, q n r S_{2}, a a c\left(6^{\prime}\right)-I b-c r$ and $q e p A$ were present in 30.8, 11.5, 3.8, 19.2 and $11.5 \%$ of those isolates, respectively. All PMQR determinants' positive isolates exhibiting 8 different genetic clones had mutations in gyrA and parC genes, and 11 carried bla genes, including 7 bla were resistant to nalidixic acid and $57.1 \%$ (8 of 14 ) of them were resistant to ciprofloxacin and levofloxacin. Our data show that there was a high prevalence of PMQR determinants in S. flexneri isolates from China.

The Journal of Antibiotics (2010) 63, 187-189; doi:10.1038/ja.2010.16; published online 5 March 2010

Keywords: plasmid; quinolone resistance; Shigella flexneri

\section{INTRODUCTION}

Shigella flexneri is a causative agent of bacillary dysentery, especially in China, and quinolones are among the most important antibacterial agents used extensively for treating this infection. ${ }^{1-3}$ Recently, quinolone resistance has been increasing markedly in these isolates. ${ }^{2,3}$ The common mechanisms for resistance to quinolone in pathogenic bacteria including $S$. flexneri are mutations in the chromosomal genes of DNA gyrase and DNA topoisomerase IV, the targets of quinolones. ${ }^{4}$ Efflux pumps and alteration in outer membrane proteins are also among the effects of chromosomal quinolone resistance. ${ }^{4}$

However, since $1998,{ }^{5}$ three kinds of plasmid-mediated quinolone resistance (PMQR) determinants have been described: Qnr, $\mathrm{AAC}\left(6^{\prime}\right)$ Ib-cr and QepA. ${ }^{6-8}$ Several families of $q n r$ ( $q n r A, q n r B$, qnrS, $q n r C$, $q n r D$ ) have been reported and each family has different subtypes. ${ }^{6,9}$ $q n r$ encoded a protein of the pentapeptide family, protecting DNA gyrase directly from quinolone inhibition. ${ }^{10} \mathrm{AAC}\left(6^{\prime}\right)$-Ib-cr is a variant of aminoglycoside acetyltransferase AAC $\left(6^{\prime}\right)$-Ib with enzymatic modification of ciprofloxacin or norfloxacin. ${ }^{7}$ qepA encoded an efflux pump belonging to the major facilitator subfamily and quinolones were the specific substrates of QepA. ${ }^{8}$

To investigate the prevalence of $\mathrm{PMQR}$ determinants in S. flexneri isolates from China, we screened the prevalence of $q n r, q e p A$ and $a a c\left(6^{\prime}\right)$ $\mathrm{Ib}-\mathrm{cr}$ genes by PCR and sequencing of positive PCR products in S. flexneri clinical isolates collected from Anhui province, China in 2005.

\section{MATERIALS AND METHODS \\ Bacteria}

A total of 26 consecutive clinical isolates of S. flexneri were collected from nine hospitals in Anhui province, China in 2005: Escherichia coli ATCC (American Type Culture Collection, Manassas, VA, USA) 25922, PMQR determinants' positive control strains and bla-positive control strains.

\section{Antimicrobial agents}

The following antimicrobial agents were used: ciprofloxacin, levofloxacin $E$-test strip (AB Biodisk, Solna, Sweden), cefotaxime, ceftazidime (National Institute for Control of Pharmaceutical and Biological Products, Beijing, China) and nalidixic acid (Sigma, St Louis, MO, USA).

Screening of qnr, qepA, aac(6')-Ib-cr, gyrA, gyrB, parC, parE and bla genes

Amplification of $q n r$, qepA and $a a c\left(6^{\prime}\right)-I b-c r$ was performed using the methods described previously, $8,9,11,12$ different primers (shown in Table 1) were used for the amplification of $q n r B$ and $q n r A$ subtypes in this study. Amplification of bla genes ( $\beta$-lactamase genes) was also performed with the methods described previously for bla $a_{\mathrm{TEM}}$, bla $\mathrm{SHV}$ and $b l a_{\mathrm{CTX}-\mathrm{M}} \cdot{ }^{13-15}$ The mutations in the quinolone resistance-determining regions of the gyrA, gyrB, parC and parE genes were amplified by PCR. ${ }^{16}$ All purified PCR products were directly sequenced using an $\mathrm{ABI} 3100$ genetic analyzer and continued using primers walking on both DNA strands. For sequence comparison, the NCBI BLAST program and facilities of the TIGR Comprehensive Microbial Resource

${ }^{1}$ Department of Infectious Diseases, The First Affiliated Hospital, Anhui Medical University, Hefei, PR China; ${ }^{2}$ School of Pharmacy, Anhui Medical University, Hefei, PR China; ${ }^{3}$ Clinical Laboratory Department, The First Affiliated Hospital, Anhui Medical University, Hefei, PR China and ${ }^{4}$ Institute of Antibiotics, Huashan Hospital, Fudan University, Shanghai, PR China

Correspondence: Professor J Li, School of Pharmacy, Anhui Medical University, 81 Meishan Road, Hefei, Anhui 230032, PR China.

E-mail: aydyxylj@126.com

Received 24 November 2009; revised 6 January 2010; accepted 5 February 2010; published online 5 March 2010 
Table 1 Primers for $q n r B$ and $q n r A$ used in this study

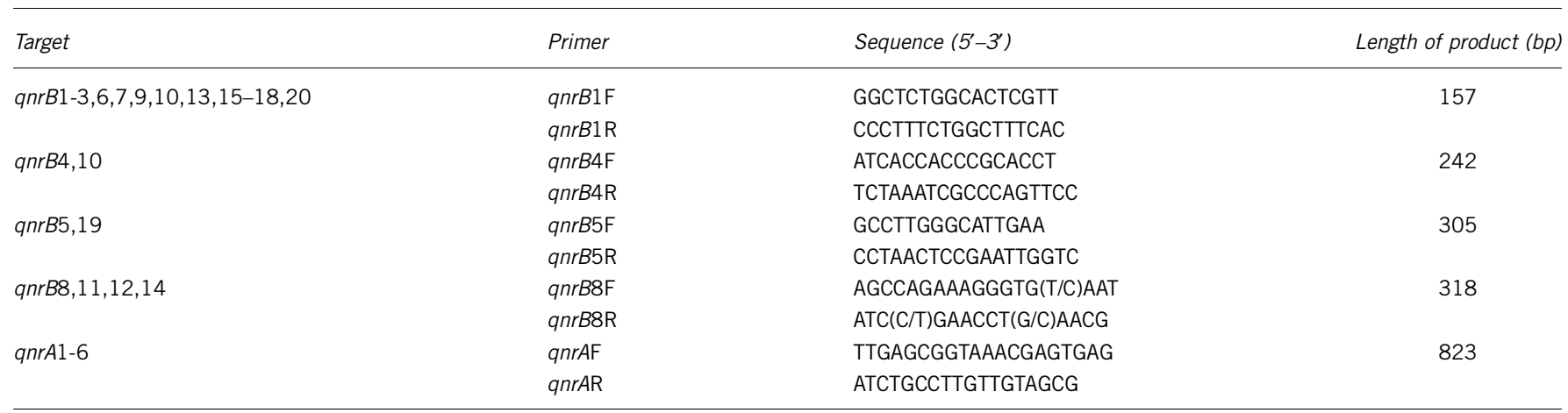

Table 2 Characteristics of the 14 PMQR determinants positive isolates

\begin{tabular}{|c|c|c|c|c|c|c|c|c|c|}
\hline \multirow[b]{2}{*}{ Isolate number } & \multirow[b]{2}{*}{ PFGE type } & \multirow[b]{2}{*}{ Resistance genes } & \multicolumn{2}{|c|}{ Mutations in QRDRs } & \multicolumn{5}{|c|}{$M I C\left(m g \digamma^{-1}\right)$} \\
\hline & & & gyrA & parC & $N A L$ & $C I P$ & LVFX & CTX & $C A Z$ \\
\hline 05ChZ60 & $A$ & qnrA1, qepA, aac $\left(6^{\prime}\right)-I b-c r$, blaTEM-1 & S83L & S80I & $>32$ & 2 & 2 & 0.125 & 0.125 \\
\hline 05ShC15 & $\mathrm{B}$ & qnrA1, blacTX-M-14 & S83L & S801 & $>32$ & 0.25 & 0.25 & $>128$ & 2 \\
\hline 05ShC18 & $\mathrm{B}$ & qnrA1, blateM-1, blacTX-M-14 & S83L & S801 & $>32$ & 0.38 & 0.5 & $>128$ & 2 \\
\hline 05ShC36 & C & qnrA1, qepA & S83L & S801 & $>32$ & 0.38 & 0.38 & $\leqslant 0.0625$ & 0.125 \\
\hline 05ShC42 & D & qnrA1, blactX-M-14 & S83L & S80I & $>32$ & 0.25 & 0.5 & $>128$ & 2 \\
\hline 05FuY15 & $E$ & qnrA1 & S83L & S801 & $>32$ & 0.38 & 0.5 & 0.125 & 1 \\
\hline 05FuY2 & $\mathrm{F}$ & qnrA1, qnrS1, bla $\mathrm{TEM}-1_{1}$, bla $\mathrm{CTX}_{\mathrm{CT}-14}$ & S83L & S801 & $>32$ & 6 & 4 & 128 & 2 \\
\hline 05FuY17 & $\mathrm{G}$ & qnrA1, qnrS1, bla & S83L & S80I & $>32$ & 4 & 8 & $>128$ & 2 \\
\hline $05 \mathrm{HN} 73$ & $E$ & qnrS1 & S83L & S80I & $>32$ & 6 & 8 & 0.125 & 2 \\
\hline 05ChY30 & $\mathrm{H}$ & 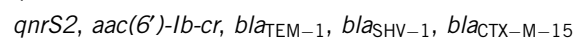 & S83L, D87N & S801 & $>32$ & $>32$ & $>32$ & 128 & 32 \\
\hline $05 \mathrm{ChY} 64$ & $\mathrm{H}$ & qepA, bla $\mathrm{TEM}-1$ & S83L, D87Y & S80I & $>32$ & $>32$ & $>32$ & $\leqslant 0.06$ & 0.125 \\
\hline 05ShC24 & 1 & $\operatorname{aac}\left(6^{\prime}\right)-I b-c r$, blaTEM-1, & S83L & S80I & $>32$ & 4 & 0.5 & 0.5 & 1 \\
\hline $05 S h C 25$ & I & $\operatorname{aac}\left(6^{\prime}\right)-l b-c r, b / a \mathrm{TEM}-1$ & S83L & S80I & $>32$ & $>32$ & 16 & 0.5 & 1 \\
\hline $05 G Z 24$ & $\mathrm{H}$ & $\operatorname{aac}\left(6^{\prime}\right)-I b-c r$, blatEM-1, blacTX-M-14 & S83L & S80I & $>32$ & $>32$ & $>32$ & 128 & 128 \\
\hline
\end{tabular}

Abbreviations: CAZ, ceftazidime; CIP, ciprofloxacin; CTX, cefotaxime; LVFX, levofloxacin; MIC, minimum inhibitory concentration; NAL, nalidixic acid; PFGE, pulsed-field gel electrophoresis; PMQR, plasmid-mediated quinolone resistance; QRDR, quinolone resistance-determining region.

(http://www.tigr.org, National Center for Biotechnology Information, Bethesda, MD, USA) were used.

\section{Antimicrobial susceptibility tests}

Minimum inhibitory concentrations (MICs) of ciprofloxacin and levofloxacin were determined using $E$-tests. Susceptibility testing for cefotaxime, ceftazidime and nalidixic acid was performed by the agar dilution method according to the guidelines recommended by the Clinical and Laboratory Standards Institute. ${ }^{17}$ E. coli ATCC 25922 was used as the quality control strain.

\section{Molecular typing of bacterial DNA}

To elucidate the clonality of PMQR determinants' positive isolates, genomic DNA of each PMQR determinants' positive strain was analyzed by pulsed-field gel electrophoresis (PFGE) in a CHEF-MAPPER apparatus (Bio-Rad Laboratories, Hercules, CA, USA) after digestion with XbaI. ${ }^{15,18}$ Genetic clones were identified according to criteria established by Tenover et al. ${ }^{18}$

\section{RESULTS AND DISCUSSION}

PMQR determinants were present in $53.8 \%$ (14 of 26) of isolates with $q n r, a a c\left(6^{\prime}\right)-I b-c r$ and qepA detected alone or in combination (Table 2). qnrA1, qnrS1, qnrS2, aac $\left(6^{\prime}\right)-I b-c r$ and $q e p A$ were present in $30.8 \%$ ( 8 of 26 ), $11.5 \%$ ( 3 of 26 ), 3.8\% ( 1 of 26 ), 19.2\% ( 5 of 26 ) and $11.5 \%$ ( 3 of 26 ) of those isolates, respectively. Five isolates carried two or three types of PMQR determinants. In 14 PMQR determinants' positive isolates, all had the mutations in gyrA (S83L and/or D87N) and parC (S80I) genes, and the ciprofloxacin MICs of these strains were relatively high, 0.25 to $>32 \mu \mathrm{g} \mathrm{ml}^{-1}$; of these, nine strains had MICs 2 to $>32 \mu \mathrm{g} \mathrm{ml}^{-1}$. A total of 11 carried bla genes (most of the strains carried more than one type), including $7 b l a_{\text {CTX-M }}(6$ $b l a_{\mathrm{CTX}-\mathrm{M}-14}$ and $\left.1 b l a_{\mathrm{CTX}-\mathrm{M}-15}\right), 9 b l a_{\mathrm{TEM}-1}$ and $1 b l a_{\mathrm{SHV}-1}$, in all 14 PMAR-positive strains simultaneously. All PMQR determinants' positive isolates were resistant to nalidixic acid and $57.1 \%$ ( 8 of 14) of them were resistant to ciprofloxacin and/or levofloxacin. Seven $b l a_{\mathrm{CTX}-\mathrm{M}}$-positive strains were resistant to cefotaxime, only two of seven were resistant to ceftazidime according to CLSI standards. ${ }^{17}$ Results of PFGE show that 14 PMQR determinants' positive isolates exhibited eight different genetic clones by Tenover et al. ${ }^{18}$ criteria.

In 2005, qnrS $S_{1}$ was first described in S. flexneri isolates from Japan. ${ }^{19}$ The qnrS1 gene has $\sim 60$ and $\sim 50 \%$ homology to the gene of qnrA and $q n r B$, respectively. There are 17 amino-acid substitutions between QnrS1 and QnrS2, but only one amino-acid substitution between QnrS1 and QnrS3. ${ }^{6} q n r C$ and $q n r D$ are novel $q n r$-like genes and encoded 221 amino-acid and 214 amino-acid pentapeptide repeat proteins, respectively, with similarities to $q n r A, q n r B$ and $q n r S .{ }^{9,11}$ $a a c\left(6^{\prime}\right)-I b-c r$ and $q e p A$ genes are the second and third most recently detected PMQR determinants differing from the $q n r$ family and are being increasingly identified worldwide among various clinical isolates 
of Enterobacteriaceae. ${ }^{7,8,12,20}$ To our knowledge, this study is the first report of $q n r A_{1}, q n r S_{2}, a a c\left(6^{\prime}\right)-I b-c r$ and $q e p A$ genes in S. flexneri (the nucleotide sequence reported herein has been registered with the EMBL database under accession nos. FJ177519, FJ177521, FJ949571, FJ842530, respectively). The emergence of PMQR determinants in $S$. flexneri may affect the use of quinolones in the treatment of dysentery, and this study provides some new and useful information.

Most of the PMQR determinants provide only low-level resistance to fluoroquinolones, but can supplement to other quinolone-resistance mechanisms, such as the selection of chromosomal mutations in the presence of a quinolone. ${ }^{4,5,7,8}$ PMAR determinants confer lowlevel resistance to quinolones with ciprofloxacin MICs $0.1-1.0 \mu \mathrm{g} \mathrm{ml}^{-1}$ in E. coli J53. For high-level ciprofloxacin- or levofloxacin-resistant strains, additional mechanisms such as target mutations and/or active efflux pump frequently coexist with PMQR determinants. ${ }^{7}$ In this study, mutations in gyrA and parC genes were identified in all PMQR determinants' positive isolates with resistance to nalidixic acid, and more than half of which were resistant to ciprofloxacin by CLSI criteria. ${ }^{17}$ S83L, D87Y mutations in gyrA and S80I in parC are the most common point mutations identified to cause quinolone resistance in several previous studies, ${ }^{4,16,21}$ and mutants with a single gyrA mutation had ciprofloxacin of MIC $0.32 \mu \mathrm{g} \mathrm{ml}^{-1}$. $^{21}$ PMQR determinants are usually associated with additional resistant determinants and have often been reported to be coassociated with ESBL (extendedspectrum $\beta$-lactamase) genes and plasmid-mediated AmpC genes. ${ }^{4}$ More than half of the PMQR determinants associated with bla genes in this study, especially $b l a_{\mathrm{CTX}-\mathrm{M}}$, which conferred coresistance to the third generation cephalosporins. CTX-M-ESBLs (especially CTX-M14) prefer hydrolyzing cefotaxime than ceftazidime and are the main type of ESBLs in Enterobacteriaceae isolates, including Shigella spp., in China. ${ }^{3,13,14}$

Results of PFGE show clonal spread in a few of these strains, which poses a serious threat to clinic. Some isolates (05ShC15/ 05ShC42 and 05FuY2/05FuY17) carry the same resistance genes, but the strains are in different PFGE types. It is possible that they were invaded by the same plasmid, which we will study in the future. Strains 05 ShC24 and 05 ShC25 are both in PFGE group I, and both carry aac $\left(6^{\prime}\right)$-Ib-cr and blaTEM-1, but are different in CIP and LVFX. There may be other forms of chromosomal resistance, such as alteration in the outer membrane proteins. ${ }^{4}$ Of three PFGE $\mathrm{H}$ types, two strains had two mutations at both 83 and 87 positions in $g y r A$, whereas one strain only had a mutation at 83 . The explanation might be that PFGE detects the molecular typing of bacterial DNA only by big DNA fragment mappers ${ }^{18}$ and those strains isolated from different hospitals.

Our data show that PMQR determinants were prevalent and $q n r A_{1}, q n r S_{2}, a a c\left(6^{\prime}\right)-I b-c r$ and $q e p A$ genes were first reported in S. flexneri from China. Most of those isolates are coresistant and clone spread exists in a few of them. It is necessary to monitor these isolates closely.

\section{ACKNOWLEDGEMENTS}

This study was supported in part by grants from the Key Technology Program of the Ministry of Education (209059), Outstanding Youth fund of Anhui province (08040106815) and from the Department of health, human research and social security of Anhui province, China.

1 Turner, S. A., Luck, S. N., Sakellaris, H., Rajakumar, K. \& Adler, B. Role of attP in integrase-mediated integration of the Shigella resistance locus pathogenicity island of Shigella flexneri. Antimicrob. Agents Chemother. 48, 1028-1031 (2004).

2 Vasilev, V., Japheth, R., Yishai, R. \& Andorn, N. Antimicrobial resistance of Shigella flexneri serotypes in Israel during a period of three years: 2000-2002. Epidemiol. Infect. 132, 1049-1054 (2004).

3 Xiong, Z., Li, T., Li, H., Xu, Y. \& Li, J. The genotype of extended-spectrum $\beta$-lactamases in Shigella flexneri isolates. Chin. J. Infect. Dis. 24, 296-300 (2006).

4 Jacoby, G. A. Mechanisms of resistance to quinolones. Clin. Infect. Dis. 15, S120-S126 (2005).

5 Martinez-Martinez, L., Pascual, A. \& Jacopy, G. A. Quinolone resistance from a transferable plasmid. Lancet 351, 197-199 (1998).

6 Jacoby, G. A. et al. qnr gene nomenclature. Antimicrob. Agents Chemother. 52, 2297-2299 (2008).

7 Robicsek, A. et al. Fluoroquinolone-modifying enzyme: a new adaptation of a common aminoglycoside acetyltransferase. Nat. Med. 12, 83-88 (2006).

8 Yamane, K. et al. New plasmid-mediated fluoroquinolone efflux pump, QepA, found in an Escherichia coli clinical isolate. Antimicrob. Agents Chemother. 51, 3354-3360 (2007).

9 Wang, M. et al. New plasmid-mediated quinolone resistance gene, anrC, found in a clinical isolate of Proteus mirabilis. Antimicrob. Agents Chemother. 53, 1892-1897 (2009).

10 Tran, J.H., Jacoby, G.A. \& Hooper, D. C. Interaction of the plasmid-encoded Quinolone resistance protein Qnr with Escherichia coli DNA gyrase. Antimicrob. Agents Chemother. 49, 118-125 (2005)

11 Cavaco, L. M., Hasman, H., Xia, S. \& Aarestrup, F. M. qnrD, a novel gene conferring transferable quinolone resistance in Salmonella enterica serovar Kentucky and Bovismorbificans strains of human origin. Antimicrob. Agents Chemother. 53, 603-608 (2009).

12 Xiong, Z. et al. Investigation of $q n r$ and $a a c\left(6^{\prime}\right)-l b-c r$ in Enterobacter cloacae isolates from Anhui Province, China. Diagn. Microbiol. Infect. Dis. 62, 457-459 (2008).

13 Xiong, Z. et al. A Klebsiella pneumoniae producing three kinds of class A $\beta$-lactamases encoded by one single plasmid isolated from a patient in Huashan Hospital, Shanghai, China. Int. J. Antimicrob. Agents 23, 262-267 (2004).

14 Xiong, Z., Li, T., Xu, Y. \& Li, J. Detection of CTX-M-14 extended-spectrum $\beta$-lactamase in Shigella sonnei isolates from China. J. Infect. 55, e125-e128 (2007).

15 Xiong, Z. et al. Investigation of extended-spectrum $\beta$-lactamase in Klebsiellae pneumoniae and Escherichia coli from China. Diagn. Microbiol. Infect. Dis. 44, 195-200 (2002).

16 Dutta, S. et al. Alteration in the GyrA subunit of DNA gyrase and the ParC subunit of topoisomerase IV in quinolone-resistant Shigella dysenteriae serotype I clinical isolates from Kolkata, India. Antimicrob. Agents Chemother. 49, 1660-1661 (2005).

17 CLSI. Performance Standards for Antimicrobial Susceptibility Testing: 17th Informational Supplement. M100-S17 (Clinical and Laboratory Standards Institute, Wayne, PA: Clinical and Laboratory Standards Institute, 2007).

18 Tenover, F. C. et al. Interpreting chromosomal DNA restriction patterns produced by pulsed-field gel electrophoresis: criteria for bacterial strain typing. J. Clin. Microbiol. 33, 2233-2239 (1995)

19 Hata, M. et al. Cloning of a novel gene for quinolone resistance from a transferable plasmid in Shigella flexneri 2b. Antimicrob. Agents Chemother. 49, 801-803 (2005).

$20 \mathrm{Ma}$, J. et al. High prevalence of plasmid-mediated quinolone resistance determinants $q n r, \operatorname{aac}\left(6^{\prime}\right)-I b-c r$, and $q e p A$ among ceftiofur-resistant Enterobacteriaceae isolates from companion and food-producing animals. Antimicob. Agents Chemother. 53, 519-524 (2009).

21 Hopoper, D. C., Wolfson, J. S., Ng, E. Y. \& Swartz, M. N. Mechanisms of action of and resistance to ciprofloxacin. Am. J. Med. 82, 12-20 (1987) 